\title{
Sex Differences and Opposite Effects of Stress on Dendritic Spine Density in the Male Versus Female Hippocampus
}

\author{
Tracey J. Shors, Chadrick Chua, and Jacqueline Falduto \\ Department of Psychology and Center for Collaborative Neuroscience, Rutgers University, Piscataway, \\ New Jersey 08854
}

\begin{abstract}
Dendritic spines are postsynaptic sites of excitatory input in the mammalian nervous system. Despite much information about their structure, their functional significance remains unknown. It has been reported that females in proestrus, when estrogen levels are elevated, have a greater density of apical dendritic spines on pyramidal neurons in area CA1 of the hippocampus than females in other stages of estrous (Woolley et al., 1990). Here we replicate these findings and in addition, show that females in proestrus have a greater density of spines in area CA1 of the hippocampus than males. Moreover, this sex difference in spine density is affected in opposite directions by stressful experience. In response to one acute stressful event of intermittent tailshocks, spine density was enhanced in the male hippocampus but reduced in the female hippocampus. The decrease in the female was observed for those that were stressed during diestrus 2 and perfused $24 \mathrm{hr}$ later during
\end{abstract}

It is well accepted that males and females can behave differently, but how much of that difference is attributable to environmental versus biological factors is a matter of debate. Under some circumstances, males and females respond in opposite directions to the same experience. For example, in response to one acute stressful experience, male rats acquire an associative learning task faster and emit more conditioned responses (Shors et al., 1992; Shors, 2001), whereas female rats exposed to the same stimulus are learning impaired, emitting fewer conditioned responses (Wood and Shors, 1998; Shors et al., 1998; Wood et al., 2001). Because there are no environmental or sociocultural factors to consider, these differences must be attributable to biological differences between the male and female. Indeed, it has been shown that the enhanced conditioning in males after exposure to the acute stressor is dependent on the presence of stress hormones glucocorticoids, whereas the impaired conditioning in females is dependent on the presence of the sex hormone estrogen (Wood and Shors, 1998; Wood et al., 2001).

Dendritic spines are potential sources of contact between many if not most excitatory neurons in the mammalian brain (Engert and Bonhoeffer, 1999; Kirov and Harris, 1999; Vanderklish et al.,

Received Jan. 12, 2001; revised May 9, 2001; accepted May 16, 2001.

This work was supported by National Institute of Mental Health Grant R01-59970, the National Alliance for Research on Schizophrenia and Depression, and the van Ameringen Foundation (T.J.S.). We thank E. Gould, P. Tanapat, J. Heal, and L. King for technical advice and E. Gould and L. D. Matzel for comments on this manuscript.

Correspondence should be addressed to T. J. Shors, Department of Psychology, Center for Collaborative Neuroscience, 152 Frelinghuysen Road, Rutgers University, Piscataway, NJ 08854-8020. E-mail: shors@rci.rutgers.edu.

Copyright (ㄷ) 2001 Society for Neuroscience $0270-6474 / 01 / 216292-06 \$ 15.00 / 0$ proestrus. The opposing effects of stress were not evident immediately after the stressor but rather occurred within $24 \mathrm{hr}$ and were evident on apical and to a lesser extent on basal dendrites of pyramidal cells in area CA1. Neither sex nor stress affected spine density on pyramidal neurons in somatosensory cortex. Sex differences in hippocampal spine density correlated with sex hormones, estradiol and testosterone, whereas stress effects on spine density were not directly associated with differences in the stress hormones, glucocorticoids. In summary, males and females have different levels of dendritic spine density in the hippocampus under unstressed conditions, and their neuronal anatomy can respond in opposite directions to the same stressful event.

Key words: estrogen; learning; fear; glutamate; corticosterone; testosterone; synapse; memory
2000). The number of actual synapses is vast, with one CA3 neuron making as many as 20,000 synapses on a CA1 pyramidal cell (Shephard and Harris, 1998). Because spines provide a means whereby neurons could become associated after sensory stimulation or other experience, it has been suggested that they may play a role in the formation of associative memories. Indeed, there are data to suggest that learning or training can affect the density of dendritic spines in several brain regions such as the cerebral cortex (Jones et al., 1997). There are also data to suggest that learning alters spine density in the hippocampus (Moser et al., 1994, 1997; Jones et al., 1997; O’Malley et al., 2000), a brain region critical for the formation of some types of memories (Solomon et al., 1986; Clark and Squire, 1998; Riedel et al., 1999; Weiss et al., 1999), including those differentially affected by stress in males versus females (Beylin and Shors, 1998; Wood et al., 2001).

The most robust modulator of spine density so far established is the sex hormone estrogen. It has been shown that exposure to estrogen either exogenously or endogenously during proestrus $(\mathrm{P})$ greatly enhances spine density in area CA1 of the hippocampus (Gould et al., 1990; Woolley et al., 1990; Woolley and McEwen, 1992, 1993). Over the 5 d estrous cycle of the rat, spine density can fluctuate as much as $30 \%$. It has not been determined whether there are sex differences in dendritic spine density in the hippocampus or elsewhere, but given the robust effects of estrogen on spine density, sex differences are likely. Here, we tested whether there are sex differences in dendritic spine density in area CA1 of the hippocampus and somatosensory cortex. Because exposure to acute stress has opposite effects on memory formation in males versus females (Shors et al., 2000) and dendritic 
spines have been implicated in learning processes, we also tested whether exposure to a stressful experience would have opposite effects on dendritic spine density in these same regions.

\section{MATERIALS AND METHODS}

Subjects and determination of stage of estrous. Sprague Dawley male and female rats (250-350 gm; $\sim 2-3$ months) were housed individually, and sexes were housed in separate rooms. Each morning (10:00-11:00 A.M.) vaginal smears were taken from virgin females for at least 2 consecutive estrous cycles, and only those with normal 4-5 d cycles, including proestrus, estrus (E), diestrus 1 (D1), and diestrus 2 (D2), were used in the study. Sterile cotton-tipped applicators were immersed in physiological saline and gently inserted into the vaginal tract to remove loose cells. Cells were rolled onto a slide (Everett, 1989). Cells were dried and fixed in $95 \% \mathrm{EtOH}$, rinsed in distilled $\mathrm{H}_{2} 0$, stained in slightly alkaline $1 \%$ aqueous filtered Toluidine blue, and rinsed in $70 \%$, and then $90 \% \mathrm{EtOH}$. Based on their vaginal cytology, rats were classified into four stages of estrous as follows: proestrus was associated with pinkish-purple staining epithelial cells with dark nuclei, estrus with masses of dark blue staining cornified cells, diestrus 1 with dark leukocytes and numerous epithelial cells, and diestrus 2 with a similar morphology but reduced numbers of epithelial cells. Relative to estrus and diestrus 1, proestrus is associated with relatively high levels of circulating estrogen (Feder, 1981; Shors et al., 1999).

Stressor procedure. On the day of stressor exposure (10:00-11:00 A.M.), smears were obtained. Immediately thereafter, cells were stained, and stage of estrous was determined. As soon as possible (within 1-2 hr), groups of male rats $(n=5)$ or females in either diestrus $2(n=5)$ or estrus $(n=5)$ were restrained and exposed to $301 \mathrm{sec}, 1 \mathrm{~mA}, 60 \mathrm{~Hz}$ shocks to the tail. Within minutes of stressor cessation (the $0 \mathrm{hr}$ stress condition in Fig. $1 A$ ), these groups of stressed rats were killed with unstressed groups of males $(n=7)$ or females in diestrus $2(n=5)$ and estrus $(n=6)$ (Fig. $1 A)$. Additional groups of males $(n=6)$ and females in diestrus $2(n=7)$ and estrus $(n=6)$ were exposed to the restraint and tail shock stressor and returned to their home cages. Twenty-four hours later, these stressed groups were removed from their home cages and killed with a group of unstressed males $(n=7)$ and groups of females that were in the same stage as the stressed females at the time of killing [i.e., $\mathrm{P}, n=7$; D2, $n=6$ ] (the $24 \mathrm{hr}$ condition in Fig. $1 B$ ). Thus, groups of females were stressed in $E$ or D2 and killed either immediately and in the same stage of estrous or $24 \mathrm{hr}$ later in the subsequent stages of D1 and $\mathrm{P}$, respectively.

Golgi method. Rats were deeply anesthetized with an overdose of Nembutal (sodium pentobarbitol) and transcardially perfused with 120 $180 \mathrm{cc}$ of $4.0 \%$ paraformaldehyde in $0.1 \mathrm{~m}$ phosphate buffer with $1.5 \%$ $(\mathrm{v} / \mathrm{v})$ picric acid. Brains were post-fixed and stored overnight. A modified version of the single-section Golgi impregnation procedure was used to process the brains (Gabbott and Somogyi, 1984). Coronal sections (100 $\mu \mathrm{m})$ were cut and immersed in baths of $3.0 \%$ potassium dichromate solution and incubated overnight. Brain sections were rinsed, mounted, coverslipped on ungelatinized glass slides with a small drop of Krazy Glue (Elmer's Products Inc., Columbus, $\mathrm{OH}$ ) on each of the four corners, and left overnight in $1.5 \%$ silver nitrate solution. Slides were then dismantled and sections were rinsed and dehydrated with ethanol $(95 \%$, then $100 \%$ ), cleared with xylene and reassembled, coverslipped with Permount, and dried.

Methods for experiment 2. In a second experiment, we replicated the opposing effects of stress on spine density $24 \mathrm{hr}$ later in males versus females that were stressed in diestrus 2 and killed in proestrus. We extended the observation of apical spines on CA1 pyramidal cells to include basal spines on the same neurons, as well as apical spines on pyramidal cells in the somatosensory cortex. In addition, we measured levels of the stress hormone corticosterone (CORT) and sex hormones estradiol and testosterone. Groups of male (stress = 6; no stress =6) and female rats in diestrus 2 (stress $=4$ ) were exposed to the stressor and killed $24 \mathrm{hr}$ later with groups of unstressed males $(n=6)$ and unstressed females in proestrus $(n=4)$. Trunk blood was collected for radioimmunoassay of hormones. Golgi impregnation was conducted as before, and the analysis was conducted blind to the experimental conditions.

Radioimmunoassay of hormone levels. As indicated, cardiac blood was collected before perfusion. Samples were immediately added to test tubes containing $0.1 \mathrm{ml}$ heparin and promptly centrifuged for $20 \mathrm{~min}$ at $3000 \mathrm{rpm}$. Plasma aliquots were stored at $-80^{\circ} \mathrm{C}$ and thawed before analysis. Circulating levels of CORT, estradiol, and testosterone were
A
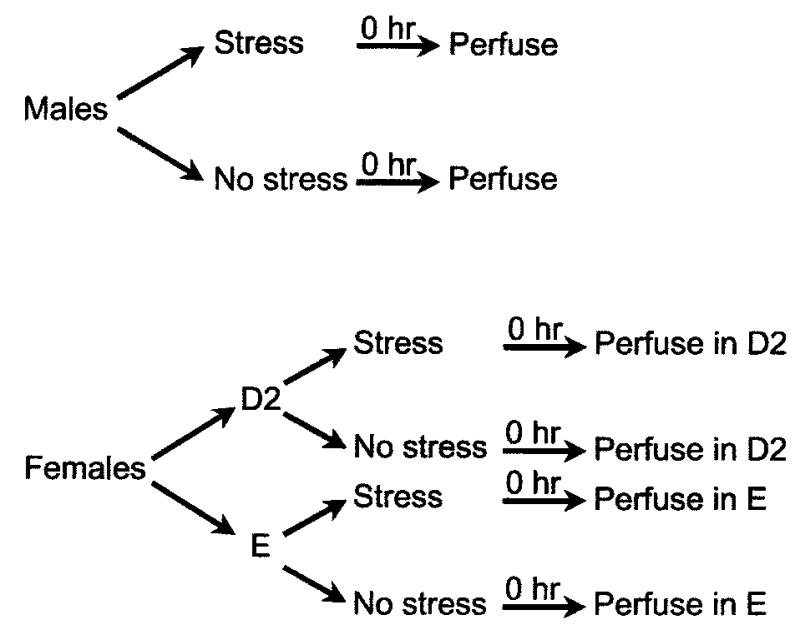

B
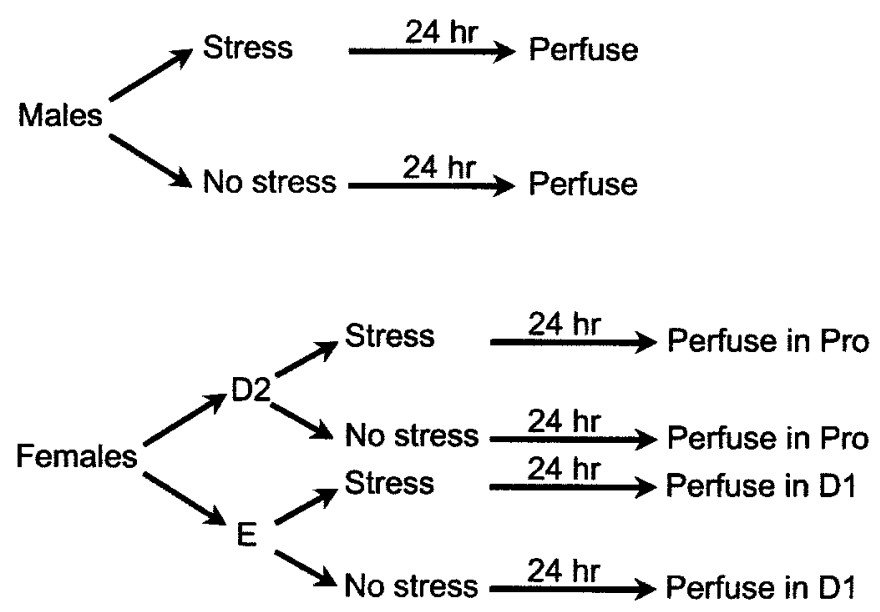

Figure 1. Schematic for design for experiment 1. Males and females in determined stages of estrous were exposed to the stressor and either immediately $(A)$ or $24 \mathrm{hr}$ later $(B)$ were perfused for determination of spine density at that time point.

measured using a solid-phase radioimmunoassay (RIA) system (Coat-ACount; Diagnostic Products). Assay sensitivity for CORT, estradiol, and testosterone were $5.7 \mathrm{ng} / \mathrm{ml}, 8 \mathrm{pg} / \mathrm{ml}$, and $4 \mathrm{ng} / \mathrm{dl}$.

Spine analysis. To be selected for quantitative analysis, the tissue had to be stained dark with Golgi impregnation that was uniform throughout the section. For an animal to be counted, it would have at least six Golgi-impregnated pyramidal neurons: (1) located within the CA1 subregion of the dorsal hippocampal formation, (2) stained and impregnated without breaks in staining along its dendrites, and (3) discernible from neighboring impregnated cells. Several guidelines were met before counting commenced on a selected neuron: (1) measurement occurred $>50 \mu \mathrm{m}$ away from the soma (a range of 50-150 $\mu \mathrm{m}$ total distance from the soma), (2) secondary and tertiary branches of apical and basal primary dendrites were located, and segments were analyzed in $10 \mu \mathrm{m}$ segments, and (3) five segments of $10 \mu \mathrm{m}$ each in the same plane of focus were chosen. In some cases, the segments were from the same branch. Counting required focusing in and out with the fine adjustment of the microscope using $1000 \times$ and oil immersion. Only those spines that were distinct from the dendritic branch were counted. For analysis of cortical neurons, at least three neurons that were: (1) located in somatosensory trunk regions and parietal association cortices (3.3-3.8 $\mathrm{mm}$ posterior to bregma; 2-3 mm lateral) (Paxinos and Watson, 1986), (2) stained and 
impregnated, and (3) easily distinguished from neighboring cells. For each cortical cell, five segments of $10 \mu \mathrm{m}$ each in the same plane of focus were chosen, and counting commenced at least $25 \mu \mathrm{m}$ from the soma on secondary and tertiary branches of apical dendrites. Spine density values are underestimates because the method does not account for spines protruding beneath or above the segment. We did not analyze for possible changes in dendritic diameter in response to either sex or stress, which could alter the appearance of spines without changing their number.

Densities of spines on five segments of a cell were averaged for a cell mean, and the six cells from each animal were averaged for an animal mean. ANOVA was conducted using the animal means as a dependent measure and stress and sex and stage of estrous at the time of perfusion as independent measures. Dependent measures included the density of apical and basal dendrites in area CA1, density on cortical neurons, and hormone levels. To evaluate group differences, post hoc analysis using Newman-Keuls was applied to significant main effects or interactions.

\section{RESULTS}

\section{Opposite effects of stress $24 \mathrm{hr}$ later in the male versus female hippocampus}

Results from the experiment addressing the effects of stress on apical dendrites in the hippocampus $24 \mathrm{hr}$ after its cessation are presented first (Figs. $1 B, 2$ ). There was a significant two-way interaction between the stress versus no stress condition and sex including the two stages of estrous in the female (diestrus 1 and proestrus $)\left(F_{(2,32)}=50.15 ; p<0.000001\right)$. Using Newman-Keuls post hoc analysis, it was determined that stressed males had a greater density of spines $(>25 \%)$ when compared with other groups in this experiment $(p<0.0005)$, including the unstressed males $(p<0.0005)$. Exposure to the stressor was also associated with changes in spine density in females, but this effect was dependent on the stage of estrous. Females that were stressed 24 hr earlier during estrus and perfused during diestrus 1 had a greater density of spines than their stage-matched controls, the unstressed females that were perfused during diestrus 1 ( $p=$ $0.01)$. Thus, the effect of stress on this group of females was enhancing and similar to that observed in the males. In contrast, females that were stressed $24 \mathrm{hr}$ earlier during diestrus 2 and perfused in proestrus had a reduced spine density $(\sim 20 \%)$ when compared with their stage-matched controls-unstressed females that were also perfused during proestrus ( $p=0.001)$. In summary, exposure to the stress had different effects on spine density that was dependent on sex and the stage of estrous in the female. There were also sex differences and effects of stage of estrous on spine density in the unstressed groups of rats. Unstressed females in proestrus had a greater density of spines than unstressed females in diestrus $1(\sim 25 \%$; $p<0.0005)$ and unstressed males $(\sim 20 \% ; p<0.005)$.

\section{Stress does not alter spine density immediately after its cessation in males or females}

Next we present data that evaluated the effects of stress on apical dendritic spine density in area CA1 within minutes of stressor cessation (Fig. 1A). Using the stress condition (stress versus no stress) and sex and stage of estrous (male versus estrus female versus diestrus 2 female) as independent variables, there was no main effect of stress $\left(F_{(1,28)}=0.95 ; p=0.34\right)$ and no interaction between stress and sex versus stages of estrous $\left(F_{(2,28)}=0.01 ; p=\right.$ $0.99)$. Thus, there was no effect of stress on dendritic spine density immediately after stressor cessation. However, there was a main effect of estrous stage and sex differences $\left(F_{(2,28)}=5.32 ; p=\right.$ $0.01)$. Females in estrus had a reduced density compared with females in diestrus $2(p<0.01)$ and males $(p<0.05)$. The mean density $(+$ SEM) was $13 / 10 \pm 0.71 \mu \mathrm{m}$ for males, $14 / 10 \pm 0.66 \mu \mathrm{m}$ for females in diestrus 2 , and $11 / 10 \pm 0.58 \mu \mathrm{m}$ for females in estrus. Although we did not statistically compare groups across the first and second parts of experiment 1 (Fig. 1, compare $A, B$ ), dendritic spine density was greater for females in diestrus 2 than in diestrus 1 and similar to those in proestrus, suggesting that estradiol levels had already begun to rise (Woolley and McEwen, 1992).

\section{Sex and stress alter dendritic spine density in area CA1 of the hippocampus but not cortex}

In the final experiment, we replicated the results of the first experiment. There was a significant interaction between the stressor condition (stress versus no stress) and sex (male versus proestrus female) on the density of apical spines in area CA1 of the hippocampus $\left(F_{(1,16)}=19.05 ; p<0.0005\right)$ (Fig. $\left.4 A\right)$. Thus, exposure to the acute stressful stimulus differentially affected apical spine density in area CA1 of the hippocampus and interacted with sex. As in the first experiment, unstressed females in proestrus had a greater density of apical dendritic spines than unstressed males $(p<0.05)$. Exposure to the stressor increased apical spine density in males relative to the unstressed males ( $p<$ 0.01 ) and decreased apical spine density in females that were stressed in diestrus 2 and perfused $24 \mathrm{hr}$ later in proestrus relative to unstressed females that were also perfused in proestrus $(p<$ $0.05)$. We extended the measurements to include the basal dendrites on CA1 pyramidal cells. Sex and stress also differentially affected the density of spines on basal dendrites in area CA1, as illustrated by the interaction $\left(F_{(1,16)}=7.16 ; p<0.05\right)$ (Fig. $\left.4 B\right)$. However, post hoc analysis revealed that only stressed males were different from stressed females, having a greater density of spines on basal dendrites $(p<0.05)$ (Fig. $4 B)$. We also evaluated the effects of stress and sex on spines on pyramidal neurons in the somatosensory cortex (Fig. $4 C$ ). In contrast to the hippocampus, neither sex $(p=0.40)$ nor stress $(p=0.72)$ altered spine density on the cortical neurons, nor was there an interaction between stress and sex on the cortical spines $(p=0.91)$.

\section{Relationship between spine density and sex and stress hormones}

With respect to corticosterone, females had higher levels than males $\left(F_{(1,16)}=18.97 ; p<0.001\right)$. Stress did not affect or differentially affect corticosterone levels in either sex $24 \mathrm{hr}$ after cessation of the stressor ( $p=0.84 ; p=0.96$, respectively) (Fig. 5A). Males had elevated levels of testosterone relative to females in proestrus $\left(F_{(1,16)}=16.08 ; p<0.001\right)$, but there was no effect of stress $(p=0.19)$ nor interaction between sex and stress on testosterone $(p=0.19)$ (Fig. $5 B)$. Females had elevated levels of estradiol relative to males $\left(F_{(1,16)}=15.76 ; p<0.001\right)$, but again, there was no effect of stress $(p=0.49)$ nor interaction between stress and sex on estradiol ( $p=0.61)$ (Fig. 5C). Across groups and conditions, spine density did not correlate with any of the hormones measured $(p>0.05)$.

\section{DISCUSSION}

Previous studies have demonstrated that spine density in area CA1 of the hippocampus fluctuates across the estrous cycle (Woolley et al., 1990; Woolley and McEwen, 1992; Woolley, 1998). The highest density occurs during proestrus, a period before ovulation when estradiol levels peak. We replicate these findings here. In addition, we present new data indicating that unstressed females in proestrus have a greater density of spines in area CA1 than unstressed males (Figs. 2, 3C,E). Moreover, we report that the density of spines in males versus females respond 


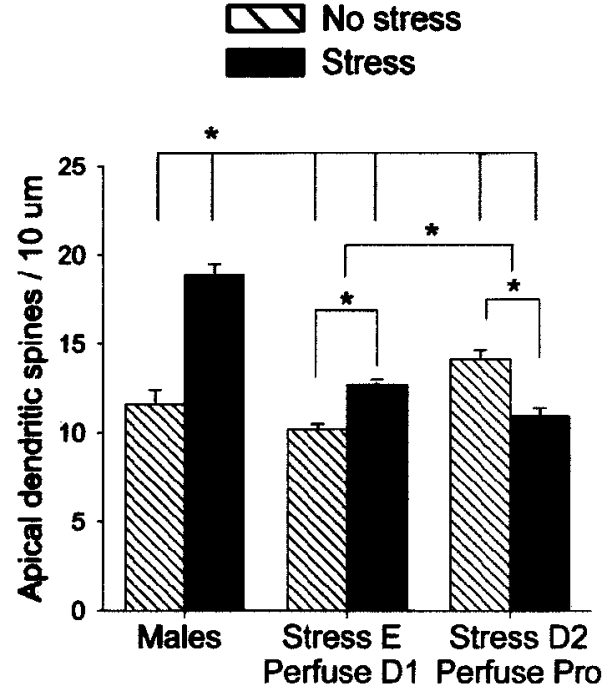

Figure 2. Opposite effects of stress on spine density in males versus females. Graph illustrates the mean $( \pm$ SEM) density of apical dendritic spines on pyramidal cells in area CA1 of the hippocampus $24 \mathrm{hr}$ after exposure to an acute stressor of brief inescapable tail stimulation. Significant differences are noted with asterisks. Under unstressed conditions, females in proestrus had a greater density of spines than males or females in diestrus 1. Exposure to the stressor increased synaptic spine density in males and decreased density in females that were stressed in diestrus 2 and perfused $24 \mathrm{hr}$ later in proestrus.

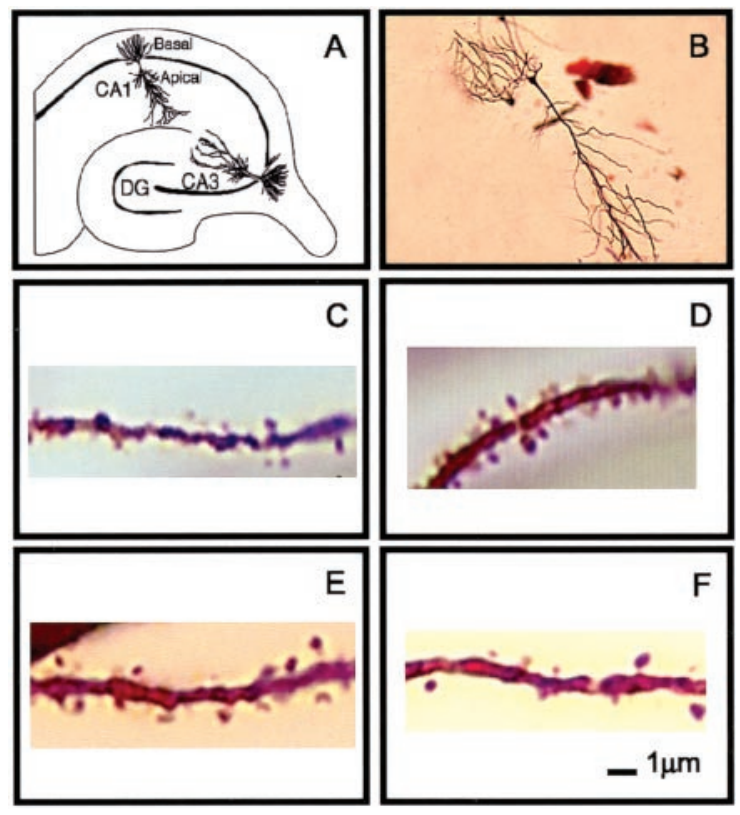

Figure 3. Golgi impregnation of apical dendrites in area CA1 of the hippocampus. $A$, A diagram of the hippocampal formation illustrating the apical and basal dendrites of CA1 pyramidal cells. $B$, Golgi-impregnated pyramidal cell, dendrite from unstressed male $(C)$, stressed male $(D)$, unstressed female in proestrus $(E)$, and female stressed during diestrus 2 and perfused $24 \mathrm{hr}$ later in proestrus $(F)$.

in opposite directions to the same stimulus. Twenty-four hours after exposure to a relatively short $(30 \mathrm{~min})$ yet fearful event of brief intermittent tailshocks, the density of spines on apical dendrites in the male hippocampus increased by as much as $30 \%$ (Figs. 2, 3C,D), whereas that of females in proestrus was reduced (Figs. 2, 3E,F). Although the effects of stress on spine density in
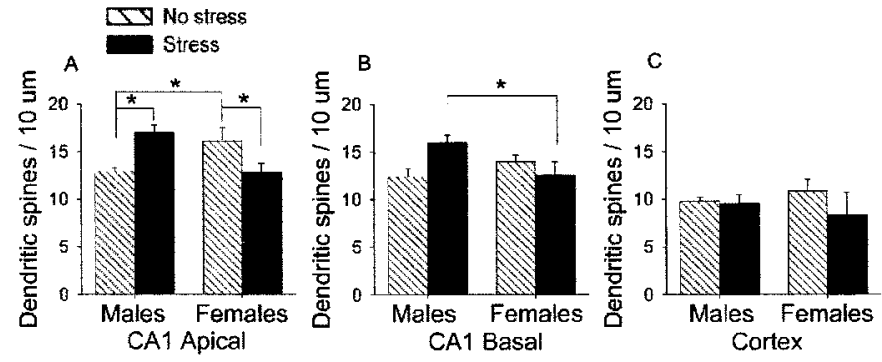

Figure 4. Effects of sex and stress on apical and basal hippocampal spines and cortical spines. Graph illustrates the mean $( \pm$ SEM) density of apical and basal dendritic spines on pyramidal cells in area CA1 of the hippocampus $24 \mathrm{hr}$ after stressor exposure. Significant differences are noted with asterisks. Exposure to the acute stressor differentially affected density of apical $(A)$ and basal $(B)$ dendritic spines on pyramidal cells of area CA1 of the male versus female hippocampus. $C$, Exposure to the stressor did not alter spine density in the cortex.

males and proestrus females were evident $24 \mathrm{hr}$ after its cessation, they were not evident within minutes of stressor cessation. Thus, although spines themselves are dynamic and motile structures (Desmond and Levy, 1998; Fischer et al., 1998), the present stress-induced changes in spine density are not rapidly induced and require some time to accumulate. Once established, stressinduced changes in spine density are evident for at least $1 \mathrm{~d}$ after cessation of the stressful event.

In the second set of studies, we replicated the opposite effects of stress $24 \mathrm{hr}$ later on apical spine density in males versus females and extended the observation to include basal dendrites of the same neurons (Fig. 4B). Although there was a significant interaction suggesting effects similar to those observed for the apical dendrites, the differences were not significant between individual stressed and unstressed groups. Thus, the effect on these dendrites is not as pronounced as that on the apical dendrites. The functional significance of this differential impact is unknown. Axons from CA3 can terminate on the apical dendrite of one neuron in CA1 and the basal dendrite of another (Amaral and Witter, 1995). However, there is a heavier commissural projection to basal dendrites of stratum oriens than apical dendrites in radiatum (Swanson et al., 1978), with corresponding differences in plasticity in these two regions (Kaibara and Leung, 1993; Cavus and Teyler, 2001). With respect to spines outside the hippocampus, we observed no effect of sex or stress on pyramidal neurons in the overlying somatosensory cortex (Fig. 4C). These results are consistent with previous reports that sex did not affect spine density in the adult visual cortex (Munoz-Cueto et al., 1991). In summary, the effects of sex and stress on spine density are not pervasive throughout the brain, and they are, instead, regionally specific.

With regard to sex and stress hormones, estrogen and corticosterone levels were enhanced in females relative to males, and testosterone was enhanced in males relative to females (Fig. 5). There was no effect of stress on corticosterone or estradiol $24 \mathrm{hr}$ after its cessation, after a stress-induced increase would have returned to baseline (Shors et al., 1999). These results indicate that the sex differences and opposite effects of stress on spine density are not determined by any one hormonal system. We have not yet manipulated sex hormones to evaluate more thoroughly their role in these effects. In addition to hormonal modulation, dendritic spine density can be modulated by changes in neurotransmission and in particular activation of glutamate receptors. For example, antagonizing NMDA receptors prevents the 


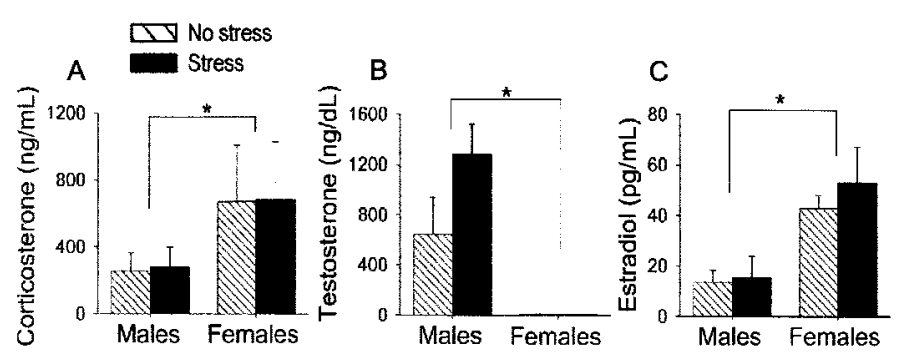

Figure 5. Effects of sex and stress on hormone levels. Blood levels of corticosterone $(A)$, testosterone $(B)$, and estradiol $(C)$ in males versus proestrus females under stressed and unstressed conditions are presented.

estrogen-induced increase in spine density in ovariectomized females (Woolley and McEwen, 1994). Others have shown that increases in spine density occur in response to AMPA-mediated activity (McKinney et al., 1999). In a related manner, exposure to the stressor used here greatly enhances AMPA receptor binding in area CA1 of the male hippocampus (Tocco et al., 1991), suggesting increases in AMPA activity as a possible mechanism for enhancing spine density after stress, at least in males. Others, however, have shown that a reduction in neuronal activity increases spine density in the hippocampus (Kirov and Harris, 1999).

\section{Contribution of estrogen to reduction in spine density after stress in proestrus females}

As noted, the reducing effect of stress on spine density was limited to females in proestrus, suggesting that females are especially sensitive to stressful experience when estrogen levels are high (Fig. 2). Because unstressed females have an enhanced density of spines in proestrus, the effect of stress in spine density in females can be interpreted in one of two ways: either exposure to a stressful experience reduces spine density in the proestrus female or exposure to the experience prevents the enhancement of spine density that occurs normally when estrogen levels are high, as during proestrus. In either case, contributions of estrous cycle to the stress-induced reduction in spine density in females suggest that the effect is dependent on the presence of estrogen.

\section{Dendritic spines and memory formation}

It has long been accepted that new dendritic spines or branches could form new synapses and thereby reinforce associations between cells or establish new ones. This capacity for connectivity is one among several means by which spines could participate in the formation of new associative memories (Cajal, 1894). Indeed, manipulations that affect learning can affect branching of dendrites (Wantanabe et al., 1992; Galea et al., 1997; Jones et al., 1997), and learning itself can affect their structure (Weiler et al., 1995; Geinisman et al., 2000). There are only a few studies to suggest that learning affects spine density, per se, at least in the hippocampus. One study reported a transient increase in spine density in the dentate gyrus after spatial learning (O'Malley et al., 2000) whereas another observed increased density on basal but not apical dendrites of CA1 after a similar training procedure (Moser et al., 1994, 1997). In an ultrastructural analysis, Geinisman et al. (2000) reported that trace eyeblink conditioning did not affect axospinous synapse number in area CA1 of the hippocampus, although measurements were limited to the apical dendrites. We are currently investigating the effects of trace conditioning on dendritic spine density on basal dendrites of those neurons.

\section{An opportunistic role for dendritic spines in the modulation of learning and memory}

From the above discussion, it is apparent that learning can alter spine density under some conditions, but whether spines are necessary for the formation of associative memories is unknown. It may be that differing levels of spine density or the availability of dendritic spines modulate learning. In fact, the opposite effects of stress on spine density in males versus proestrus females reported here are very similar in effect to those of stress on learning in males versus proestrus females (Wood et al., 2001). Using hippocampal-dependent trace conditioning as a measure of performance, we have shown that exposure to the acute tailshock stressor greatly facilitates conditioning in the male rat $24 \mathrm{hr}$ later but reduces performance in the proestrus female (Beylin and Shors, 1998; Shors, 1998, 2001; Shors et al., 2000; Wood et al., 2001). Moreover, we have shown that unstressed females in proestrus acquire the conditioned response faster than do unstressed males. Thus, under these various conditions, memory formation is positively related to dendritic spine density in area CA1 of the hippocampus. That is, stressed males have a greater density of spines than unstressed males, and they condition more. Also, unstressed females in proestrus have a greater density of spines than unstressed males or females in other stages of estrous and they condition more. Finally, unstressed females in proestrus have a greater density of spines than stressed females in proestrus and they condition more, again when trained $24 \mathrm{hr}$ after the stressor in proestrus. Given these data, it could be proposed that an increase in spine density (either endogenously through high levels of estrogen in females or exogenously via stressor exposure in males) "primes" the nervous system for efficient learning. The presence of those spines can then be used for a more rapid formation of new memories should the opportunity arise. In this scenario, the important point is that the presence of spines is opportunistic rather than deterministic.

In summary, we present data illustrating sex differences in dendritic spine density in select regions of the brain with females in proestrus having a greater density than males. In addition, we demonstrate that dendritic spines are especially sensitive to acute stressful experience and respond in opposite directions to the same stimulus simply as a matter of sex differences.

\section{REFERENCES}

Amaral DG, Witter MP (1995) Hippocampal formation. In: The rat nervous system (Paxinos G, ed), pp 443-493. San Diego: Academic.

Beylin AV, Shors TJ (1998) Stress enhances excitatory trace eyeblink conditioning and opposes acquisition of inhibitory conditioning. Behav Neurosci 112:1327-1338.

Cajal RC (1894) New ideas on the structure of the nervous system in man and invertebrates (English translation: 1990). London: MIT.

Cavus I, Teyler TJ (2001) NMDA receptor-independent LTP in basal versus apical dendrites of CA1 pyramidal cells in rat hippocampal slice. Hippocampus 8:373-379.

Clark RE, Squire LR (1998) Classical conditioning and brain systems: the role of awareness. Science 280:77-81.

Desmond NL, Levy WB (1998) Free postsynaptic densities in the hippocampus of the female rat. NeuroReport 9:1975-1979.

Engert F, Bonhoeffer T (1999) Dendritic spine changes associated with hippocampal long-term synaptic plasticity. Nature 399:66-70.

Everett BJ (1989) Neurobiology of reproduction in the female rat: monographs on endocrinology. Berlin: Springer.

Feder HH (1981) Estrous cyclicity in mammals. In: Neuroendocrinology of reproduction: physiology and behavior (Adler NT, ed), pp 280-293. New York: Plenum.

Fischer M, Kaech S, Knutti D, Matus A (1998) Rapid actin-based plasticity in dendritic spines. Neuron 20:847-854.

Gabbott PL, Somogyi J (1984) The "single" section Golgi-impregnation procedure: methodological description. J Neurosci Methods 11:221-230.

Galea LAM, McEwen BS, Tanapat P, Deak T, Spencer RL, Dhabhar FS 
(1997) Sex differences in dendritic atrophy of CA3 pyramidal neurons in response to chronic restraint stress. Neuroscience 81: 689-697.

Geinisman Y, Disterhoft JF, Gundersen JG, McEchron MD, Persina IS, Power JM, Van der Zee EA, West MJ (2000) Remodeling of hippocampal synapses after hippocampal-dependent associative learning. J Comp Neurol 417:49-59.

Gould E, Woolley CS, Frankfurt M, McEwen BS (1990) Gonadal steroids regulate dendritic spine density in hippocampal pyramidal cells in adulthood. J Neurosci 10:1286-1291.

Jones TA, Klintsova AY, Kilman VL, Siervaag AM, Greenough WT (1997) Induction of multiple synapses by experience in the visual cortex of adult rats. Neurobiol Learn Mem 68:13-20.

Kaibara T, Leung LS (1993) Basal versus apical dendritic long-term potentiation of commissural afferents to hippocampal CA1: a currentsource density study. J Neurosci 13:2391-2404.

Kirov SA, Harris KM (1999) Dendrites are more spiny on mature hippocampal neurons when synapses are inactivated. Nat Neurosci 2:878-883.

McKinney RA, Capogna M, Durr R, Gahwiler BH, Thompson SM (1999) Miniature synaptic events maintain dendritic spines via AMPA receptor activation. Nat Neurosci 2: 44-49.

Moser MB, Trommald M, Andersen P (1994) An increase in dendritic spine density on hippocampal CA1 pyramidal cells following spatial learning in adult rats suggests formation of new synapses. Proc Natl Acad Sci USA 91:12673-12675.

Moser MB, Trommald M, Egeland T, Andersen P (1997) Spatial training in a complex environment and isolation alter the spine distribution differentially in rat CA1 pyramidal cells. J Comp Neurol 380:373-381.

Munoz-Cueto JA, Garcia-Segura LM, Ruiz-Marcos A (1991) Regional sex differences in spine density along the apical shaft of visual cortex pyramids during postnatal development. Brain Res 540:41-47.

O'Malley AOC, Murphy KJ, Regan CM (2000) Transient spine density increases in the mid-molecular layer of hippocampal dentate gyrus accompany consolidation of a spatial learning task in the rodent. Neuroscience 99:229-232.

Paxinos G, Watson C (1986) The rat brain in stereotaxic coordinates. San Diego: Academic.

Riedel G, Micheau J, Lam AGM, Roloff EVL, Martin SJ, Bridge H, deHoz L, Poeschel B, McCulloch J, Morris RGM (1999) Reversible neural activation reveals hippocampal participation in several memory processes. Nat Neurosci 2:898-906.

Shephard GMG, Harris KM (1998) Three-dimensional structure and composition of CA3/CA1 axons in rat hippocampal slices: implications for presynaptic connectivity and compartmentalization. J Neurosci 18:8300-8310.

Shors TJ (1998) Stress and sex effects on associative learning: for better or for worse. The Neuroscientist 4: 353-364.

Shors TJ (2001) Acute stress rapidly and persistently enhances memory formation in the male rat. Neurobiol Learn Mem 75:10-29.

Shors TJ, Weiss C, Thompson RF (1992) Stress-induced facilitation of classical conditioning. Science 257:537-539.
Shors TJ, Lewczyk C, Paczynski M, Mathew PR, Pickett J (1998) Stages of estrous mediate the stress-induced impairment of associative learning in the male rat. NeuroReport 9:419-423.

Shors TJ, Pickett J, Wood GE, Paczynski M (1999) Acute stress enhances estrogen levels in the female rat. Stress 3:163-171.

Shors TJ, Beylin AV, Wood GE, Gould E (2000) The modulation of Pavlovian memory. Behav Brain Res 110:39-52.

Solomon PR, van der Schaaf ER, Weisz D, Thompson RF (1986) Hippocampus and trace conditioning of the rabbit's classically conditioned nictitating membrane response. Neuroscience 100:729-744.

Swanson LW, Wyss JM, Cowan WM (1978) An autoradiographic study of the organization of intrahippocampal association pathways in the rat. J Comp Neurol 181:681-716.

Tocco G, Shors TJ, Baudry M, Thompson RF (1991) Selective increase of AMPA binding to the AMPA/quisqualate receptor in the hippocampus in response to acute stress. Brain Res 559:168-171.

Vanderklish PW, Krushel LA, Holst BH, Gally JA, Corssin KL, Edelman GM (2000) Marking synaptic activity in dendritic spines with calpain substrate exhibiting fluorescence resonance energy transfer. Proc Natl Acad Sci USA 97:2253-2258.

Wantanabe Y, Gould E, McEwen BS (1992) Stress induces atrophy of apical dendrites of hippocampal pyramidal neurons. Brain Res 588:341-345.

Weiler IJ, Hawrylak N, Greenough WT (1995) Morphogenesis in memory formation: synaptic and cellular mechanisms. Behav Brain Res $66: 1-6$.

Weiss C, Bouwmeester H, Power JM, Disterhoft JF (1999) Hippocampal lesions prevent trace eyeblink conditioning in the freely moving rat. Behav Brain Res 99:123-132.

Wood GE, Shors TJ (1998) Stress facilitates classical conditioning in males but impairs conditioning in females through activational influences of ovarian hormones. Proc Natl Acad Sci USA 95:4066-4071.

Wood GE, Beylin AV, Shors TJ (2001) The contribution of adrenal and reproductive hormones to the opposing effects of stress on trace conditioning in males versus females. Behav Neurosci 115:175-187.

Woolley C (1998) Estrogen-mediated structural and functional synaptic plasticity in the female rat hippocampus. Horm Behav 34:140-148.

Woolley CS, McEwen BS (1992) Estradiol mediates fluctuation in hippocampal synapse density during the estrous cycle in the adult rat. J Neurosci 12:2549-2554.

Woolley CS, McEwen BS (1993) Roles of estradiol and progesterone in regulation of hippocampal dendritic spine density during the estrous cycle in the rat. J Comp Neurol 336:293-306.

Woolley CS, McEwen BS (1994) Estradiol regulates hippocampal dendritic spine density via an $N$-methyl-D-aspartate receptor-dependent mechanism. J Neurosci 14:7680-7687.

Woolley CS, Gould E, Frankfurt M, McEwen BS (1990) Naturally occurring fluctuations in dendritic spine density on adult hippocampal pyramidal neurons. J Neurosci 10:4035-4039. 